\title{
The effects of low-intensity blood flow restricted exercise compared with conventional resistance training on the clinical outcomes of active UK military personnel following a 3-week in-patient rehabilitation programme: protocol for a randomized controlled feasibility study
}

Peter Ladlow ${ }^{1,2^{*}} \mathbb{D}$, Russell J. Coppack ${ }^{1,2}$, Shreshth Dharm-Datta ${ }^{1}$, Dean Conway ${ }^{1}$, Edward Sellon ${ }^{3}$, Stephen D. Patterson ${ }^{4}$ and Alexander N. Bennett ${ }^{1,5}$

\begin{abstract}
Background: A challenge for rehabilitation practitioners lies in designing optimal exercise programmes that facilitate musculoskeletal (MSK) adaptations whilst simultaneously accommodating biological healing and the safe loading of an injured limb. A growing body of evidence supports the use of resistance training at a reduced load in combination with blood flow restriction (BFR) to enhance hypertrophic and strength responses in skeletal muscle. In-patient rehabilitation has a long tradition in the UK Military, however, the efficacy of low intensity (LI) BFR training has not been tested in this rehabilitation setting. The aims of this study are to determine (1) the feasibility of a randomised controlled trial (RCT) investigating LI-BFR training in a residential, multidisciplinary treatment programme and (2) provide preliminary data describing the within and between-group treatment effects of a LI-BFR intervention and a conventional resistance training group in military personnel.

Methods: This is a single-blind randomised controlled feasibility study. A minimum of 28 lower-limb injured UK military personnel, aged 18 to 50 years, attending rehabilitation at the UK Defence Medical Rehabilitation Centre (DMRC) will be recruited into the study. After completion of baseline measurements, participants will be randomised in a 1:1 ratio to receive 3 weeks (15 days) of intensive multidisciplinary team (MDT) in-patient rehabilitation. Group 1 will receive conventional resistance training 3 days per week. Group 2 will perform twice daily LI-BFR training. Both groups will also undertake the same common elements of the existing MDT programme. Repeat follow-up assessments will be undertaken upon completion of treatment. Group 2 participants will be asked to rate their pain response to LI-BFR training every five sessions.

(Continued on next page)
\end{abstract}

\footnotetext{
* Correspondence: DMRC-ResearchScientist2@mod.uk

${ }^{1}$ Academic Department of Military Rehabilitation, Defence Medical

Rehabilitation Centre (DMRC), Headley Court, Epsom, Surrey, UK

${ }^{2}$ Department for Health, University of Bath, Bath, UK

Full list of author information is available at the end of the article
} 


\begin{abstract}
(Continued from previous page)
Discussion: The results will provide information on the feasibility of a full-scale RCT. Recommendations for an adequately powered study to determine the efficacy of LI-BFR training during in-patient rehabilitation can then be made. The study may also provide insights into the potential effectiveness of LI-BFR training as a novel exercise modality to induce muscle adaptations in the absence of high mechanical loading of the lower-limb.
\end{abstract}

Trial registration: ISRCTN Reference: ISRCTN 63585315 dated 25 April 2017.

Keywords: Blood flow restriction, Musculoskeletal rehabilitation, Lower-limb, Muscle, Strength, Hypertrophy, Pain

\section{Background}

The maintenance of adequate skeletal muscle is crucial for maintaining the ability to undertake activities of daily living, ambulation, falls avoidance and general health [1]. Disuse of skeletal muscle, often associated with musculoskeletal (MSK) injury, can lead to relatively rapid and progressive atrophy; shortening of muscle fibres, decreased oxidative capacity, and reduced muscle compliance [2]. It is widely acknowledged that muscle atrophy can prolong the duration of MSK rehabilitation, increase the cost to health care providers and prevent optimal recovery [3]. Thus, strategies to increase or maintain muscle tissue across the lifespan are crucial for overall health and quality of life.

The goal of the surgical and rehabilitative team focuses on the safe return of a patient to their previous level of function. MSK rehabilitation can be considered in terms of the appropriate integration and progression of the following broad exercise components: endurance, flexibility, proprioception, balance, joint and soft tissue mobility, speed and power [4]. Strength training is most closely associated with improvements in functional ability during rehabilitation [5]. Therefore, maximising the potential for adaptations in muscle strength is a crucial factor in the progression of any MSK exercise rehabilitation programme. A significant challenge lies in designing optimal rehabilitation programs that facilitate both neurological and muscular adaptations whilst accommodating biological healing and patient safety [4]. Historically, it has been widely accepted that to elicit significant gains in muscle hypertrophy and strength requires loads equivalent to at least $70 \%$ of an individual's 1 repetition maximum (1RM) for a given movement $[6,7]$. For people undergoing musculoskeletal injury rehabilitation, heavy-load resistance training can be contraindicated [2] or they are limited by their symptomatic impairment, including pain and immobility, to attain the recommended heavier-loads [8]. Therefore, patients with MSK injuries are often advised to reduce their training load, potentially limiting the desired muscular response to treatment.

In recent years, research demonstrates that the use of blood flow restriction (BFR) combined with low-load resistance exercise (20-40\% 1RM) can enhance the morphology and strength response in human muscle tissue [9]. BFR is typically achieved via a pressurised cuff [10], tourniquet [11] or elastic banding [12]. The external pressure applied to the proximal portion of the upper or lower extremities should be low enough to maintain partial arterial inflow into the muscle, but high enough to occlude venous return from the muscle [13]. During periods of immobilisation, the application of BFR alone has been shown to reduce muscular atrophy [14]. However, to optimise muscular development, BFR must be combined with an exercise stimulus (aerobic conditioning or resistance training), with the greatest muscle strength and morphological responses achieved when BFR is combined with resistance training [2].

When supervised by experienced practitioners, lowintensity BFR (LI-BFR) has been shown to be a safe and effective tool to improve strength and function in athletes [15], the elderly [16, 17] healthy adults [18] and during MSK rehabilitation [19-24]. Additional benefits reported with LI-BFR training, is the potential for increases in muscle hypertrophy and strength in muscles located proximal to the applied pressure (i.e. muscles not direct under BFR) as a result of pre-fatigue of the muscles below the cuff [25]. It is possible that this additional muscle stimulus proximal to the cuff (e.g. in the Gluteus Maximus muscle during a squat or leg press) may further enhance physical function and accelerate progression during MSK rehabilitation. Adverse events to acute sessions of LI-BFR have been reported and primarily include delayed onset of muscle soreness, numbness, fainting/dizziness and bruising [26]. There have also been case study reports of rhabdomyolysis $[27,28]$ and retinal occlusion [29] in the literature. However, when appropriately supervised, LI-BFR is widely acknowledged as a safe mode of exercise in healthy adults [30].

It is proposed that the metabolic stress associated with BFR and the mechanical tension of the load lifted act synergistically to mediate numerous secondary mechanisms, all of which stimulate autocrine and/or paracrine actions to facilitate muscle growth [31]. These proposed mechanisms include muscle cell swelling [32], elevated systemic hormone production [33, 34], intramuscular anabolic/anti-catabolic signalling [35-37], increased fast twitch fibre recruitment [38] and the production of reactive oxygen species (ROS) $[39,40]$ and its variants, including i) nitric oxide for its 
influence on vascular responses $[41,42]$ and ii) some heat shock proteins [36, 39]. However, in the absence of research demonstrating a causal link, any suggested associations between BFR training and subsequent muscle growth are purely speculative.

There is increasing evidence for the practical and beneficial use of BFR training as a clinical MSK rehabilitation tool [43]. Any intervention that speeds the progression of MSK rehabilitation, whilst exercising at lower relative training loads, is of interest not only to the rehabilitation and sports medicine communities, but the wider community health services. In military populations, the majority of injuries occur in the lower limb [44]. In a cohort of 6608 British Army recruits, during a 26-week period of initial military training, the overall incidence of musculoskeletal injuries was $48.6 \%$ [45]. There is a large economic and operational cost associated with lower-limb MSK injury. Soldiers injured during basic training, field exercise, sport etc. may be unable to deploy on operations, whilst soldiers injured during deployment may not be fit to return to active duty [44]. The Centre for Lower-Limb Rehabilitation at the UK Defence Medical Rehabilitation Centre (DMRC),
Headley Court routinely treats and manages a large variety of lower-limb musculoskeletal disorders (See Fig. 1 for a diagrammatic model of the rehabilitation pathway). These typically include, but are not limited to, overuse injuries (e.g. patellofemoral pain, tendinopathy, early osteoarthritis, and exertional lower-limb pain), post-surgical injuries (e.g. soft-tissue and ligamentous reconstruction), bone fractures, and hip and groin pain.

Of particular interest, in relation to the UK military model of exercise rehabilitation (Fig. 1), is the evidence of muscular hypertrophy and strength demonstrated with high frequency (twice a day) BFR training in as little as 6 days [46] and 12 days [47] of training. The effect of this novel training method is yet to be explored across the UK Defence Medical Services (DMS). Therefore, the purpose of this study is to assess the feasibility of LI-BFR training in a heterogeneous group of lower-limb injured military personnel, under the conditions provided during a 3-week intensive residential rehabilitation centre. This includes measuring the hypertrophic and strength response but also reporting any potential adverse events, monitoring compliance and the pain response over time and whether

Throughout the entire rehabilitation pathway increasing functional mobility and reducing pain are primary goals. This will promote the prospect of a faster return to military duties, improve patient well-being and reduce care costs to the UK MOD.

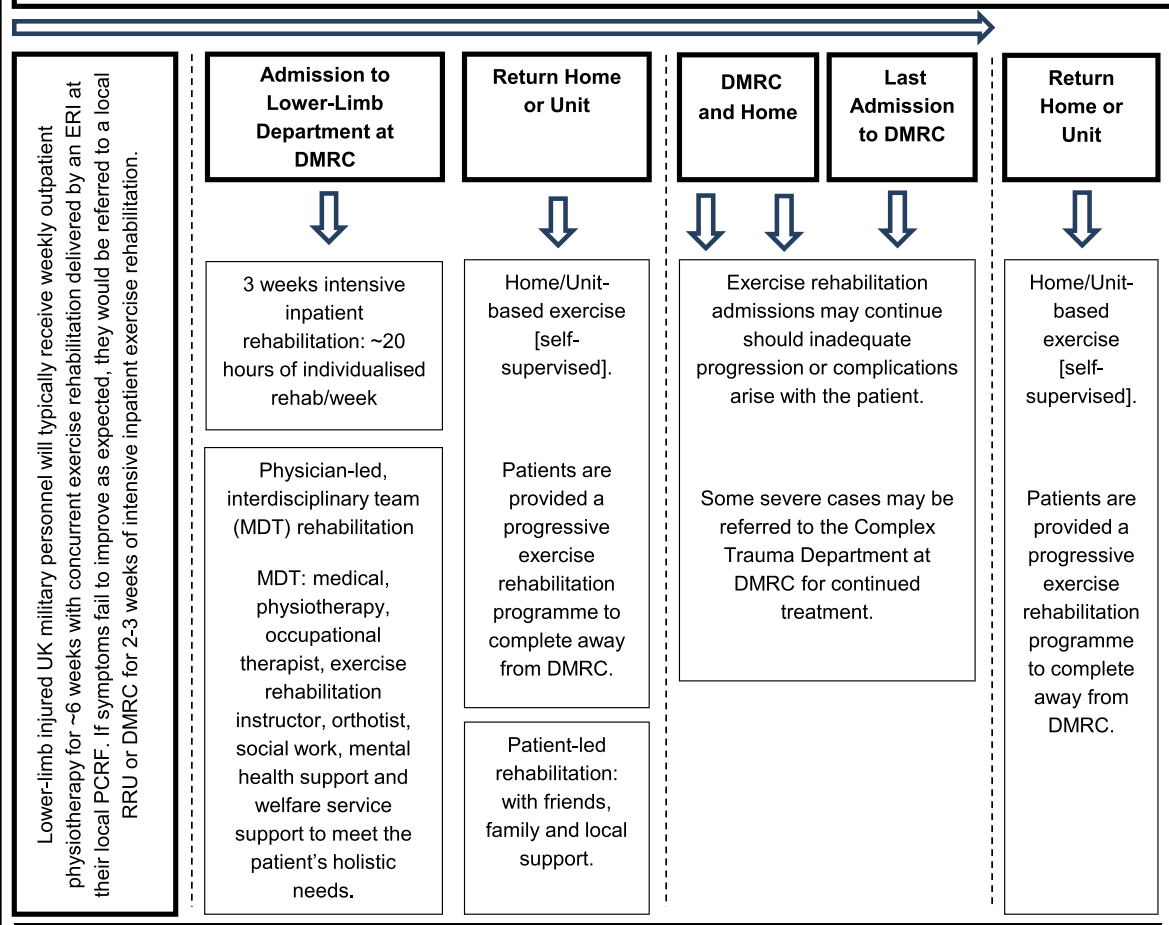

Abbreviations: $\mathrm{MOD}=$ Ministry of Defence, $\mathrm{ERI}=$ Exercise Rehabilitation Instructor, $\mathrm{PCRF}=$ Primary Care Rehabilitation Facility, RRU = Regional Rehabilitation Unit, DMRC = Defence Medical Rehabilitation Centre, MDT = Multidisciplinary team. 
frequent daily use of this clinical tool is feasible in a busy MDT clinical rehabilitation setting where other potentially conflicting clinical priorities exist. This protocol describes the design and analysis plan for a randomised controlled feasibility study.

\section{Methods/design}

The primary aim of this study is:

1. To assess the acceptability, feasibility and adverse events associated with implementing a high-frequency LI-BFR intervention in a 3-week intensive residential MDT rehabilitation setting.

2. To assess the feasibility of a future definitive RCT by assessing participant eligibility, monitoring recruitment and retention rates, group allocation acceptance and adherence to the intervention.

The secondary aim is to compare the effects of LI-BFR training against conventional resistance training on crosssectional area (CSA) and volume of the quadriceps and hamstring muscle groups and muscle strength in UK military personnel undergoing lower-limb injury rehabilitation. Changes in relevant musculoskeletal variables of treatment routinely measured as part of the standard UK military rehabilitation care pathway will also be assessed. This includes walk/run assessment, balance, pain perceptions and compliance to the exercise rehabilitation programme.

\section{Study design}

This is a parallel group, two-arm, assessor-blinded randomised controlled feasibility study. It is a two (group) by two (time) repeated measures design. Outcome measurements will be assessed at baseline and 3-weeks. The study protocol has been developed in accordance with the Standard Protocol Items: Recommendations for Interventional Trials (SPIRIT) guidelines [48]. The overall study design is illustrated in Fig. 2.

\section{Setting}

The study will be conducted at a specialist UK military rehabilitation centre.

\section{Ethics}

The study was reviewed and approved by the UK Ministry of Defence (MOD) research ethics committee (study reference protocol number: 442/MODREC/13). Any requirement for protocol modifications will be submitted for authorisation to the MOD research ethics committee.

\section{Study participants}

We will recruit a minimum of 28 participants aged 1850 years admitted for treatment to the Centre for Lower-
Limb Rehabilitation (Defence Medical Rehabilitation Centre (DMRC), Headley Court, UK) with a MSK injury of the lower limb. Table 1 describes the inclusion and exclusion criteria. These criteria are designed to recruit a heterogeneous group of lower-limb injured patients who are able to engage in load bearing conventional resistance training, but do not have a functional status allowing a return to full operational military duties. A significant majority of patients admitted to DMRC for rehabilitation are male. In a time limited' period allocated for data collection, we could not predict the number of females available for recruitment into the study. Therefore, because the primary purpose of this study is to assess the feasibility and not the effectiveness of the LI-BFR intervention, for ease of administration and logistics, we chose to recruit males only. If feasible, any future full-scale RCT will recruit both male and female participants.

\section{Randomisation and blinding}

Potential participants will be referred from their parent military unit by a physiotherapist or medical officer. Prior to admission to DMRC, the case records of individual patients scheduled for a lower-limb rehabilitation course will be reviewed by a specialist rehabilitation consultant (SDD). Those who meet the preliminary inclusion criteria will be contacted via telephone by a member of the research team to discuss their possible inclusion in the study. Potential participants will be sent an information pack consisting of the patient information sheet (PIS) and an accompanying consent form. Upon admission to DMRC Headley Court, potential participants will undertake a comprehensive musculoskeletal examination by a specialist consultant and experienced musculoskeletal physiotherapist where a secondary screening will confirm the patient's eligibility to enter the study. Participants meeting the eligibility criteria, who have read and understood the PIS and volunteer to participate will return a signed informed consent form, before being randomly assigned to one of the two study groups. A block randomisation method will be used to randomise participants into groups that result in equal sample sizes. Our decision to employ a simple form of block randomisation is because (a) we want to ensure an equal number of participants are assigned to each group during a finite, time-limited period for data collection, (b) we already have a homogenous participant group with standardised prognostic factors, and (c) we will not be undertaking formal statistical testing. A plain language statement will inform participants that they have an equal chance of receiving the LI-BFR or conventional resistance training intervention. A sealed envelope will be opened to reveal group allocation by an independent administrator not involved in the recruitment, treatment or assessment of study outcomes. Group allocation will be documented and communicated to 


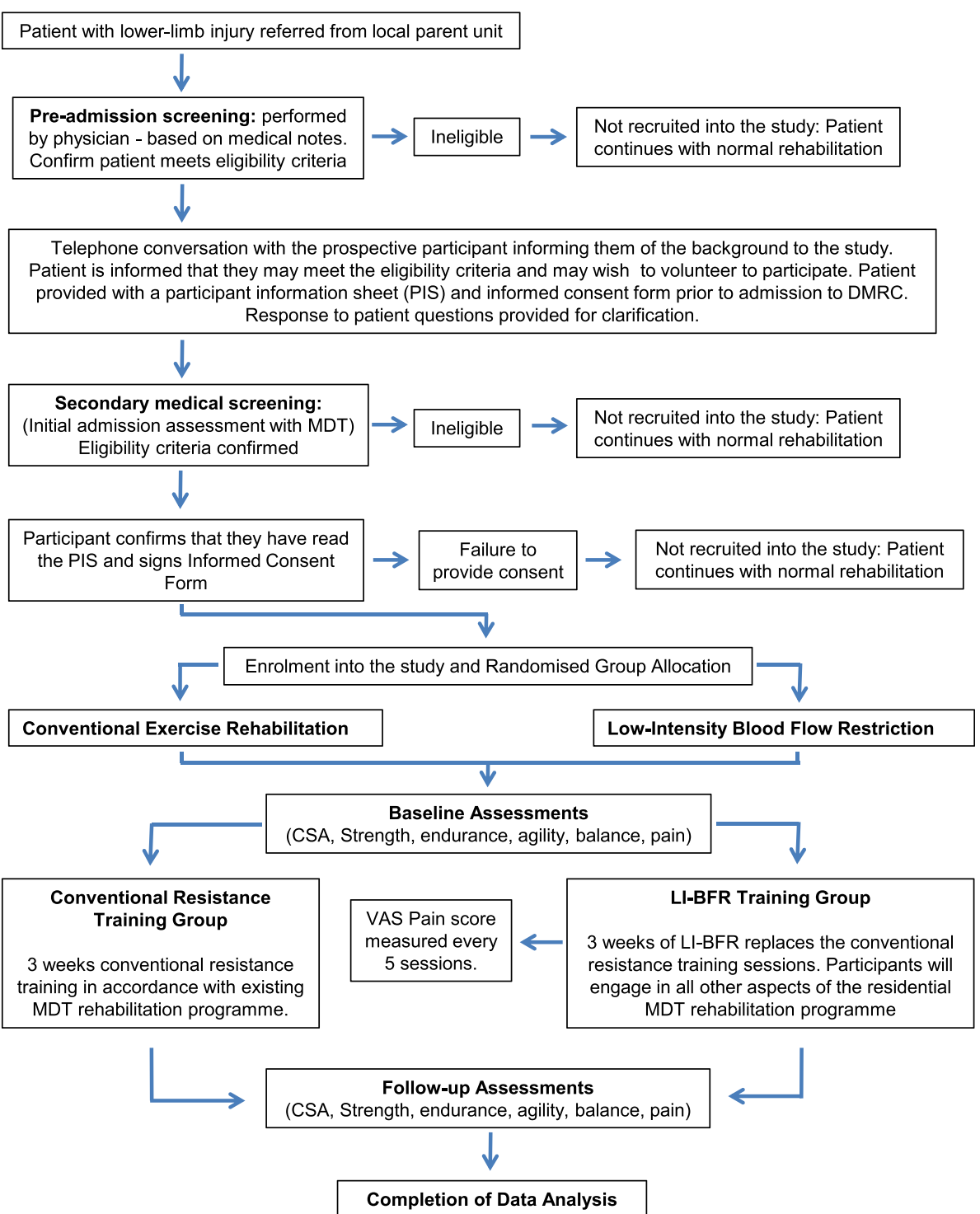

Fig. 2 Study design

the supervising therapists by the independent administrator. Prior to the study, all treating staff will have received a briefing on the randomisation process and specific intervention for each treatment group, in line with the study protocol. It is not possible to blind participants to treatment allocation in this study. The clinical staff supervising both groups will be, by necessity, un-blinded. We will use trained blinded outcome assessors to measure and record the outcome scores in this study. A diagrammatic description of the study design can be found in Fig. 2 .

\section{Combined LI-BFR and resistance training protocol—current guidelines}

Scott et al. [9] has provided evidence-based guidelines on optimal muscle hypertrophy and strength responses to LI-BFR training. This includes using cuff widths $(\sim 6$ to $13.5 \mathrm{~cm})$ for the legs and resistance training at 50 to $80 \%$ of occlusion pressure taken at rest; a detailed description of the technique used to establish occlusion pressure is provided in a later section (LI-BFR Group). BFR can be used with low-intensity exercise $(\sim 20-40 \%$ of $1 \mathrm{RM})$, utilising 50 to 80 repetitions per exercise, with occlusion maintained during inter-repetition rest periods of 30 to $45 \mathrm{~s}$. Both single and multi-joint exercises can provide benefit and clinical populations are advised to complete two to three training sessions per week; however, training twice each day with BFR is possible. These guidelines will form the foundations of our LI-BFR training protocol. 
Table 1 Study inclusion and exclusion criteria

Inclusion criteria
1. Male
2. 18 to 50 years of age
3. Serving regular UK Armed Forces personnel
4. Lower limb injury (e.g. patellofemoral pain, ACL reconstruction, ankle
injury, projectile/blast related injury)
5. Referred to Defence Medical Rehabilitation Centre (DMRC), Headley
Court for treatment

6. Present with a level of function enabling engagement in conventional load bearing exercise rehabilitation confirmed by clinical assessment findings and recent training history.

7. Unable to return to active duty due to physical impairment and occupational limitations (e.g. medical downgrading).

Exclusion Criteria

1. Female

2. History of cardiovascular disease (hypertension, peripheral vascular disease, thrombosis/embolism, ischaemic heart disease, myocardial infarction)

3. History of the following musculoskeletal disorders: rheumatoid arthritis, avascular necrosis or osteonecrosis, severe osteoarthritis

4. History of the following neurological disorders: Peripheral neuropathy, Alzheimer's disease, amyotrophic lateral sclerosis, multiple sclerosis, Parkinson's disease, stroke, mild or severe traumatic brain injury

5. Chronic or relapsing/remitting gastrointestinal disorders such as inflammatory bowel diseases, irritable bowel syndrome or gastrointestinal infections within 28 days of screening.

6. Acute viral or bacterial upper or lower respiratory infection at screening

7. Moderate or severe chronic obstructive pulmonary disease (COPD)

8. Amputation to the lower or upper extremity

9. Known or suspected lower limb chronic exertional compartment syndrome (CECS) (tourniquet raises intra-compartmental muscle pressure)

10. Achilles or patella tendinopathy (slow heavy resistance or eccentric exercise programme prescribed as evidence-based for confirmed tendinopathy diagnosis)

11. ACL surgery within the last 4 weeks

12. Surgical insertion of metal components in lower limbs (may affect MRI results)

13. History of any of the following conditions or disorders not previously listed: diabetes, fibromyalgia, active cancer, severe obesity (i.e., body mass index greater than $35 \mathrm{~kg} / \mathrm{m}^{2}$ ), diagnosed mental illness (e.g. PTSD, depression, anxiety)

14. Current or previous use of any drugs known to influence muscle mass or performance within previous 6 months

15. Elevated risk of unexplained fainting or dizzy spells during physical activity/exercise that causes loss of balance

\section{Generic lower-limb rehabilitation intervention}

The total duration of treatment is 3 weeks, utilising 15-days of specific MDT exercise rehabilitation (Monday to Friday). There is no follow-up period, as the aim is to assess the feasibility and effects of the intervention(s) during the period of in-patient rehabilitation. A summary of the DMRC lower-limb MDT rehabilitation programme and treatment components is provided at Fig. 1 and Table 2, respectively. This generic treatment approach has been described elsewhere by Coppack et al. [49]. All participants will receive individualised programmes focussing on improving range of motion, balance, aerobic conditioning, manual therapy and education sessions. The generic MDT programme will be common to both groups with only the resistance training or LI-BFR intervention individualised to each participant dependent on group allocation. Additional information regarding each of these components is provided below.

\section{Stretching and range of motion exercise}

Static and active stretching and foam-roller techniques will be employed to maintain the range of motion (ROM) required for optimal function. This forms part of routine clinical practice within lower-limb rehabilitation in the UK military.

\section{Neuromuscular control and functional balance exercise}

Balance and proprioceptive exercises will be included to restore deficits and re-establish neuro-motor control. Progression will be applied by increasing the complexity and difficulty of the exercise, by reducing the base of support, adding dynamic movements on unstable surfaces and increasing the range through which the movement is performed. Support for neuromuscular training in lower-limb rehabilitation has been reported in the literature [50].

\section{Aerobic exercise}

Participants will undertake light to moderate aerobic conditioning over the intervention period. In addition to the general health benefits conferred by aerobic exercise, moderate joint loading has been shown to be beneficial for joint health because of mechanosensitive chondroprotective pathways [51]. No study has described the optimal dose of aerobic exercise for patients undergoing lower-limb rehabilitation in terms of intensity, volume and duration. In this study, the supervising ERI will determine the nature of aerobic exercise (walking, cycling, swimming, cross-trainer) and progression in intensity based on individual examination finings and patient response to exercise.

\section{Manual therapy}

Manual therapy techniques will be used to modify the quality and range of motion of soft tissue structures, and assist with pain relief. The manual therapy intervention will be prescribed individually for each participant on the basis of the physical examination findings, from a list of techniques including, trigger point massage, passive joint mobilisation, distraction and sustained stretches [49]. These techniques are commonly used in the management of injured military personnel at DMRC and delivered by their respective MSK physiotherapist. 
Table 2 Components of generic in-patient rehabilitation programme

\begin{tabular}{|c|c|c|c|}
\hline $\begin{array}{l}\text { Treatment } \\
\text { modality }\end{array}$ & Treatment content & Treatment goals & $\begin{array}{l}\text { Typical number of } \\
\text { sessions per week }\end{array}$ \\
\hline $\begin{array}{l}\text { Individualised } \\
\text { Exercise: led by ERI } \\
\text { (45-60 min) }\end{array}$ & $\begin{array}{l}\text { Strengthening exercises, active range of motion exercises, } \\
\text { functional balance drills, gait drills, progressive } \\
\text { coordination drills, non-weight-bearing aerobic/ } \\
\text { endurance exercise }\end{array}$ & $\begin{array}{l}\text { Restore strength of major muscle groups of the } \\
\text { lower-limb, improve core strength, increase joint } \\
\text { range of motion, improve balance and } \\
\text { neuro-muscular control, and improve } \\
\text { muscle endurance. }\end{array}$ & $3-4$ \\
\hline $\begin{array}{l}\text { Individualised } \\
\text { physiotherapy } \\
\text { (30-60 min) }\end{array}$ & $\begin{array}{l}\text { Manual therapy techniques, muscle activation and timing } \\
\text { patterns, active and passive range of motion exercises, } \\
\text { advice on home exercise, gait re-education training }\end{array}$ & $\begin{array}{l}\text { Improve quality and timing of movement, } \\
\text { improve muscle strength, reduce pain, increase } \\
\text { joint range of motion, induce relaxation, } \\
\text { promote normal walking gait. }\end{array}$ & $1-3$ \\
\hline $\begin{array}{l}\text { Group Exercise: } \\
\text { led by ERI } \\
\text { ( } 45-60 \mathrm{~min})\end{array}$ & $\begin{array}{l}\text { Group based circuit training that primarily involves high } \\
\text { repetition muscular strengthening exercises targeting the } \\
\text { whole body. May also include minor team games, } \\
\text { recreational therapy, foam rolling, stretching, motor } \\
\text { control, running re-education, cv }\end{array}$ & $\begin{array}{l}\text { The same as the Individual exercise sessions, } \\
\text { but also the promotion of group cohesion and } \\
\text { social support }\end{array}$ & 12 \\
\hline $\begin{array}{l}\text { Hydrotherapy/ } \\
\text { swimming } \\
\text { (30/45 min) }\end{array}$ & $\begin{array}{l}\text { Non-weight-bearing aerobic exercise, strengthening } \\
\text { exercises, active range of motion exercises, self-paced } \\
\text { recreational swimming, progressive/assisted } \\
\text { weight-bearing exercise and activity }\end{array}$ & $\begin{array}{l}\text { Improve muscle strength, improve aerobic } \\
\text { capacity, increase joint range of motion, improve } \\
\text { confidence in weight bearing, induce relaxation, } \\
\text { and promote enjoyment and fun. }\end{array}$ & $\begin{array}{l}1 \text { hydro } \\
3 \text { swim }\end{array}$ \\
\hline $\begin{array}{l}\text { Individualised } \\
\text { occupational } \\
\text { therapy session } \\
\text { (30-60 min) }\end{array}$ & $\begin{array}{l}\text { Relaxation techniques, postural re-education, cognitive } \\
\text { behavioural therapy techniques, self-help coping } \\
\text { strategies, pain management. }\end{array}$ & $\begin{array}{l}\text { Induce relaxation, promote behavioural change, } \\
\text { control pain, correct/improve poor posture }\end{array}$ & $0-3$ \\
\hline $\begin{array}{l}\text { Patient education } \\
\text { (60 min) }\end{array}$ & $\begin{array}{l}\text { Coping with pain, benefits of exercise, joint protection, } \\
\text { anatomy and pathology of their lower-limb injury, } \\
\text { nutrition. }\end{array}$ & $\begin{array}{l}\text { Goal setting, activity modification, reduction of } \\
\text { pain, promote behavioural change, weight } \\
\text { management, improve knowledge of treatment } \\
\text { options, improve ability to relax, improve } \\
\text { knowledge of self-help techniques }\end{array}$ & 4 \\
\hline
\end{tabular}

\section{Education}

Educating the patient on factors surrounding their treatment and the importance of regular exercise is a key component of the rehabilitation process at DMRC to optimise patient adherence to home or work-based exercise programmes. Education and advice will be a focus of the intervention and will include information on diagnosis and aetiology of their injury, rationale for treatment, the benefits of exercise, joint protection and activity modification strategies, pain management, coping with acts of daily living (sitting, driving, sleeping, work) and the importance of increasing physical activity levels in everyday life [49]. Unsupervised homebased prescription of BFR has not been investigated and therefore not recommended in the UK Defence best practice guidelines. Instead, the LIBFR group will be prescribed a conventional resistance training programme to perform upon discharge from the rehabilitation centre.

\section{Intervention group 1-LIBFR group \\ Determining limb occlusion pressure}

The participant's limb occlusion pressure will be determined during a one-off procedure prior to commencing the LI-BFR training programme. The participant lies in a semi-recumbent supine position on a treatment couch and a contoured $66 \times 10 \mathrm{~cm}$ or $90 \times 10 \mathrm{~cm}$ width blood pressure cuff (Schuco TourniCuff, Schuco International, Watford, UK) is placed around the most proximal part of each thigh. The length of the cuff $(60$ or $90 \mathrm{~cm})$ will be selected based on the participant's thigh girth, thereby ensuring sufficient overlap of the inflatable regions of the cuff to provide occlusion to the lower-limb. The cuff size will be recorded and then used for the entirety of that participant's LI-BFR training programme. The posterior tibial or dorsalis pedis pulse is found with a MD2 vascular Doppler probe (Huntleigh Healthcare Ltd., Cardiff, UK). The wide contoured tourniquet will rapidly be inflated using a PTSii portable tourniquet system (Delfi Medical Innovations, Vancouver, Canada) to a pressure of $250 \mathrm{mmHg}$ [52] so that the audible pulse is lost. If the pulse is not lost at $250 \mathrm{mmHg}$, then the cuff will be inflated in increments of $10 \mathrm{mmHg}$ until the pulse is abolished. The cuff will be deflated in increments of $10 \mathrm{mmHg}$ until the pulse is found again. This provides an estimate to the nearest $10 \mathrm{mmHg}$. The cuff will then be fully deflated. After a 30 s rest, the cuff will be inflated to the estimate pressure $+10 \mathrm{mmHg}$. It will then be deflated more slowly in increments of $5 \mathrm{mmHg}$, so the occlusion pressure can be determined to the nearest $\pm 5 \mathrm{mmHg}$. $60 \%$ of this limb occlusion pressure is calculated to be used as the tourniquet pressure during the LI-BFR intervention [9]. Subsequent limb blood occlusion pressure assessments will be performed on day 7 and the final training day to measure potential changes in occlusion pressure over time. Any measured changes in pressure 
on day 7 will not influence the cuff pressure used during the trial.

\section{LI-BFR exercise protocol}

Participants will perform low intensity resistance training combined with blood flow restriction using two exercises in sequence: (1) bilateral leg press using a Leg Press Machine (Pulse Fitness, Congleton, UK), and (2) bilateral knee extensions using a Leg Extension Machine (Pulse Fitness, Congleton, UK) (see Fig. 3).

Prior to exercise, each participant will undergo a standardised 5 min progressive warm-up on a stationary bike (Wattbike Ltd., Nottingham, UK). Wide contoured blood pressure cuffs will then be placed around the most proximal part of each thigh and inflated using a PTSii portable tourniquet system to $60 \%$ occlusion pressure. Participants will then perform 4 sets of 30,15, 15 and 15 repetitions (75 repetitions in total) at $30 \%$ of their predicted $1 \mathrm{RM}$, assessed during their 5RM muscle strength assessments with an inter-set interval of $30 \mathrm{~s}$ (see secondary outcome measures below for a detailed description of the 5RM muscle strength assessment protocol). To ensure consistency of lifting between patients, a metronome is set at $60 \mathrm{bpm}$, with $1 \mathrm{~s}$ for the completion of the concentric phase, no pause followed by a $1 \mathrm{~s}$ eccentric phase of the lift (1:0:1 tempo).

The inflation pressure will be maintained for the duration of the exercise and then deflated for $3 \mathrm{~min}$ to allow the participant a small recovery period and to move to the next exercise/equipment station. It will then be re-inflated to the target $60 \%$ occlusion pressure and the second exercise station (leg extension) will commence. Therefore, the length of time induced to restricted blood flow will be
4 min per exercise and 8 min per training session. Training will be performed twice daily, in the morning (between 08:00 and 09:30) and afternoon (between 14:00 and 15:30) from Monday to Thursday and once on Friday morning. Over the 15 days of rehabilitation, MDT clinical assessments will be carried out on the first and last day of an admission. This allows a maximum of 23 LI-BFR training sessions over a total period of 13 treatment days. Daily LI-BFR sessions will always be separated by interludes of at least $5 \mathrm{~h}$. Assuming patients adapt over the 3 week residential programme, the $1 \mathrm{RM}$ is expected to increase and therefore even when exercising at 30\% 1RM, we would expect to increase the weight lifted by small increments (e.g. $2.5 \mathrm{~kg}$ increase per week). Any increase in weight lifted will be at the discretion of the exercise rehabilitation instructor (ERI) and participant, and training load (the number of repetitions and load lifted) for each session will be monitored and recorded accordingly.

\section{Intervention 2 conventional resistance training}

Participants will engage in conventional load bearing resistance training, typically consisting of four sets of three exercises (deadlift, back squat and lunges) performed three times per week. A gradual and timely exercise progression is determined by the ERI based upon participant feedback, re-assessment and individual response to training. It should be noted that despite an abundance of information on the implementation of strength and conditioning principles with healthy participants, investigation regarding the application of these principles in rehabilitation programmes is lacking [53]. Therefore, a relatively conservative initial dosage is chosen that should allow a short period of adaptation whilst controlling for pain, thereby promoting

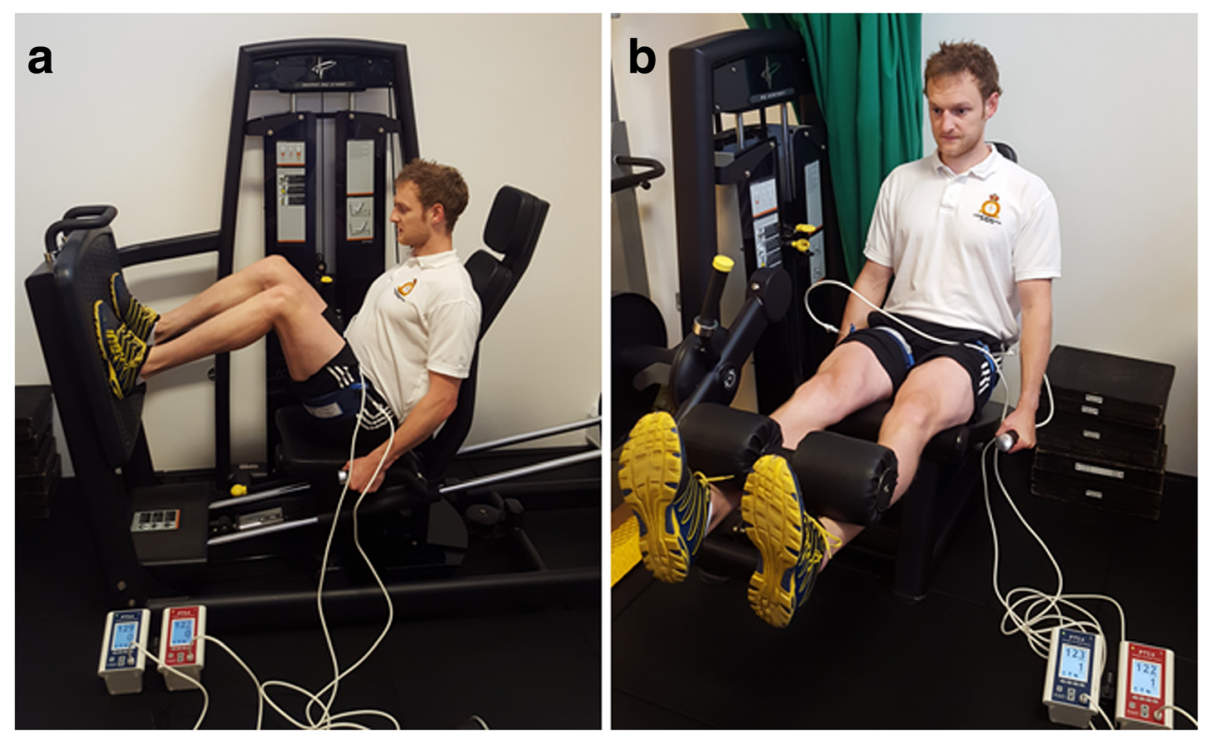

Fig. 3 Low intensity blood flow restriction (LI-BFR) exercises: a leg press, b knee extension 
exercise adherence. Patients will be educated on correct movement patterns before loading to volitional fatigue. Repetitions per set are typically six to eight and tailored to the individual needs of the patient with rest intervals between each set approximately $3 \mathrm{~min}$. The dosage for strengthening exercises in this protocol aims to meet the ongoing challenge of designing treatment programmes that facilitate neurological and muscular adaptations whilst concurrently accommodating biological healing, recovery, and the safety of the patient. The justification for the initial dosage of four sets of six to eight repetitions takes account of the evidence suggesting pain provoked by exercise has been shown to reduce adherence to exercise in rehabilitation programmes [54]. The load lifted is a reflection of their best effort taking into account each individual's respective limitations due to injury.

\section{Outcome measures}

All outcome measures are to be assessed at baseline and upon completion of 3 weeks in-patient rehabilitation. Pain response and training load will be recorded over several time points during the BFR intervention (Fig. 2). The authors acknowledge that due to the likely interference effect of a pain response, performing a 'maximum effort' physical task in a lower-limb injured cohort is unlikely to yield a true measure of MSK performance. It is more accurate to describe outcome scores as providing an 'indication' of participant performance and progression. We will highlight this as a potential weakness in our study but feel this is a challenge in the measurement of muscle force/strength in all MSK injury research. All outcomes measured will be implemented and recorded based upon a best effort at the time of assessment.

\section{Descriptive data}

Personal and demographic characteristics including age, stature, body mass, body mass index (BMI), gender, duration of symptoms, previous injuries, previous treatments, medication use, military occupation, duration of military service, smoking and drinking habits will be obtained during the initial participant assessment or obtained via electronic medical notes using the Defence Medical Information Compatibility Program (DMICP).

\section{Primary outcome measure: feasibility and acceptability of LI-BFR intervention}

The main focus of this study is feasibility and acceptability for recruitment, retention and measurement of the LI-BFR intervention. Recruitment rates will be measured as the rate of eligible participants invited and consenting into the study. Acceptability of allocation/randomisation procedures will be assessed by examining the reasons for drop-out in any discontinuing participants and by comparing attrition rates between groups. Session adherence rates will be recorded to provide a measure of compliance with the intervention. Strengths, weaknesses and safety of LI-BFR intervention will be assessed by qualitative interviews with the project supervisor, lead exercise rehabilitation instructor, participant feedback and examination of any adverse event reports. We will also aim to provide an estimate of the recommended sample-size for a fully-powered future RCT [55].

\section{Secondary outcome measure: muscle cross sectional area (CSA) and volume}

Thigh muscle cross sectional area (CSA) $\left(\mathrm{cm}^{2}\right)$ and volume $\left(\mathrm{cm}^{3}\right)$ will be assessed prior to and 1 day following the subject's rehabilitation training programme, using magnetic resonance imaging (MRI) with a GE Sigma scanner $1.5 \mathrm{~T}$ (General Electric, Wisconsin, USA), in accordance with the method previously described by Abe et al. [56]. A T1-weighted, spin-echo, axial plane sequence will be obtained with continuous transverse images from the greater trochanter to the lateral condyle of the femur with a $1.0 \mathrm{~cm}$ slice thickness and no inter-slice gap. If this distance exceeds $50 \mathrm{~cm}$, two separate sequence acquisitions will be required with a triglyceride skin marker used for sequence co-registration. The MRI data will be transferred onto a study laptop computer for analysis by a UK Defence Consultant Radiologist, using specially designed image analysis software (TomoVision Inc., Montreal, Canada) [57]. For each slice on the injured limb, quadriceps and hamstring muscle compartment CSA $\left(\mathrm{cm}^{2}\right)$ will be measured and muscle compartment volumes calculated $\left(\mathrm{cm}^{3}\right)$. In participants with bilateral pathology, one leg will be randomly selected for muscle analysis. The coefficient of variation $(\mathrm{CV})$ for this measurement technique has been demonstrated to be less than $1 \%$ [56]. Repeat MRI assessments will be performed $24 \mathrm{~h}$ after the participant's final LI-BFR training session.

\section{Secondary outcome measure: muscle strength}

Unilateral muscle strength will be assessed using a dynamic 5 RM test performed on the knee extension and leg press machines (Pulse Fitness, Congleton, UK). Following a general warm-up on a stationary bike for $5 \mathrm{~min}$, subjects will perform a 10-repetition warm-up on the knee extension and leg press respectively. An initial resistance is set by the supervising ERI based upon the result of a clinical assessment, pain intensity and participant feedback. The resistance is then adjusted and test repeated until the participant is unable to complete five repetitions. The baseline $5 R M$ is then recorded by the supervising ERI. Subjects will have 3 min rest between each attempt. This procedure follows established and widely used guidelines [58]. 


\section{Secondary outcome measure: functional performance assessment}

Functional ability will be assessed using the following standardised outcome measures used in the current best-practice care pathway at DMRC. These tests will be conducted and recorded by an experienced supervising therapist.

\section{Isometric muscle strength proximal to the cuff}

Unilateral isometric muscle strength will be measured at the start and end of the 3 weeks. Measurements will be taken using a wireless digital microFET2 hand-held dynamometer (Hoggan Scientific LLC, Drapper, UT, USA) for hip extension only (see Fig. 4). Isometric hip extension strength will measure muscular adaptations proximal to the cuff. Participants will be tested on a clinical examination couch using procedures often applied in the clinical setting [59]. This test was chosen as isometric loading induces less stress on the musculoskeletal system than eccentric loading ('break-test'), which is a key consideration when testing individuals with a physical injury [60]. A long lever arm will be utilised during the test to ensure the tester's strength exceeds the isometric force applied by the participant. The examiner will apply resistance in a fixed position whilst the participant exerts a $5 \mathrm{~s}$ isometric maximal voluntary contraction (MVC) against the dynamometer and the examiner. Participants will perform four consecutive attempts with a 30 s recovery between attempts. Strength measures will be reported as Newtons $(\mathrm{N})$. The highest value will be used for analysis purposes. Good interrater reliability (ICC 0.76-0.79) and low test-retest variation $(<10 \%)$ has been demonstrated for the HHD measurement technique in measuring isometric muscle strength [59].

\section{Multi-stage locomotion test (MLFT)}

The objective of this test is to assess the participant's maximal walk/run distance $[61,62]$. The test requires the participant to walk/run on a $20 \mathrm{~m}$ track at gradually increasing speeds until they are unable to continue due to an increase in symptoms. Speed is controlled by pacedauditory cues accompanied by recorded verbal instructions. The test will be terminated once a patient fails three consecutive attempts at reaching the designated marker at the sound of the audible cue. Total distance covered in metres will be recorded.

\section{Figure of 8 test}

This test measures the participant's agility and acceleration/ deceleration ability on a flat surface. Within the limitations of their injury, each participant will be required to complete 3 laps of a figure of 8 walk/run at maximal speed, in accordance with established guidelines [63].

\section{Y-balance test}

This test developed by Plisky et al., [64] assesses lower-body balance and flexibility using the Y-Balance test kit ${ }^{\circ}$. Standing through a single supporting limb on the test kit, the participant will reach with the free limb as far as possible along three lines positioned in anterior, posteromedial and posterolateral directions on each leg (see Fig. 5a-c). The test is currently used as a measure of postural control in patients undergoing UK military lower-limb rehabilitation.

\section{Pain perception}

It is recognised that BFR training can cause mild muscle discomfort. A visual analogue scale (VAS) will be used to measure pain intensity. The VAS uses a $100 \mathrm{~mm}$ horizontal line anchored by the terms 'no pain' (0) and 'worst possible pain' (100). The VAS response format has shown good internal consistency, is easy to understand, is in wide clinical use, and has been sufficiently evaluated in clinical trials [65]. It is useful in this prospective study of twice daily LI-BFR training sessions to record pain scores in the injured limb. This will be

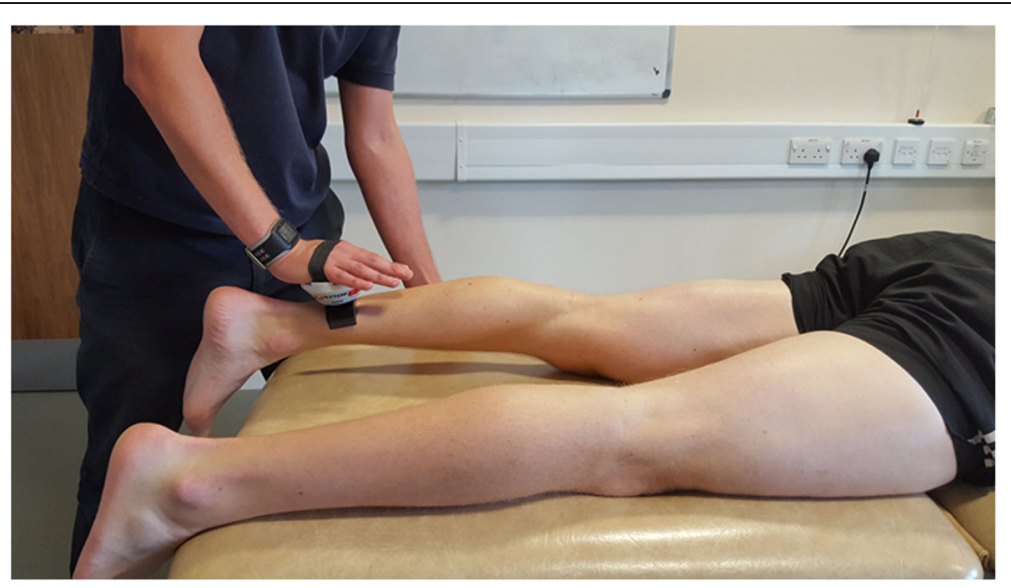

Fig. 4 Hip extension strength measure using microFET-2 hand-held dynamometer (HHD) 
recorded immediately prior to starting the exercise, during the exercise and then $5 \mathrm{~min}$ post-exercise. This will be repeated every five BFR training sessions to monitor how pain response changes over time to the BFR intervention.

\section{Sample size}

As this is a pilot-feasibility study we will not perform a formal sample size calculation determined by statistical assumptions and tests. Sample size recommendations for pilot randomised controlled trials will be followed [66] and will aim for a minimum of 12 participants in each study arm providing full data. Given the time constraints for data collection, we intend to recruit 14 participants into each treatment group (i.e. total sample size of 28). We consider this will provide sufficient data to adequately address the aims of this feasibility study and provide useful information on key issues such as recruitment, retention and acceptability of the LI-BFR intervention. This pragmatic preliminary sample will inform a power analysis for a full-scale RCT.

\section{Statistical analyses}

Data generated from this pilot study will help inform a future fully-powered RCT by testing the study procedures. We will not use feasibility trial data to formally test for between-group differences, and the analysis will be of a descriptive nature. Therefore, no statistical analyses will be performed for the pilot data. Descriptive statistics (mean, standard deviation (SD), counts (percentage)) will be used to summarise eligibility, consent, randomisation, adverse events, retention, completion and intervention adherence rates. Description of participant demographic and baseline characteristics will be compared and simple tabulation of this pilot data will be presented. The 95\% confidence intervals will be calculated to inform a sample size for a future definitive RCT. All participants will be included in the analysis. This statistical analysis of the pilot data will be exploratory only as our sample size will not allow for a definitive analysis. We will recommend progression to a full study application if minimum criteria are reached in key feasibility aims and objectives. These criteria will include a minimum $80 \%$ of target participant recruitment over a 6-month period, and a minimum $80 \%$ completion of the LI-BFR intervention and outcome measurement.

\section{Adverse events}

All clinical and research staff will receive a brief detailing the procedures for identifying and reporting safety issues including the use of project adverse events forms. Information on any unexpected adverse events deemed to be related to study participation will be collected and reported to the chief investigator within $24 \mathrm{~h}$ of its occurrence. Reporting of safety incidents will be duplicated using existing DMRC clinical health and safety reporting procedures and in accordance with the principles of good clinical practice (GCP). It is not anticipated that there will be any risk to study participants.

\section{Discussion}

Optimising the recovery of UK Military personnel suffering MSK injury is of critical importance and has been highlighted as a priority for research by the UK Defence Medical Services. The premise that the use of BFR combined with low-load resistance exercise can enhance the strength response in human muscle tissue may have implications for the rate of recovery in patients undergoing injury rehabilitation. We describe the rationale and design of a feasibility study for the introduction of an LI-BFR intervention into a residential, MDT rehabilitation programme for military patients suffering a variety of

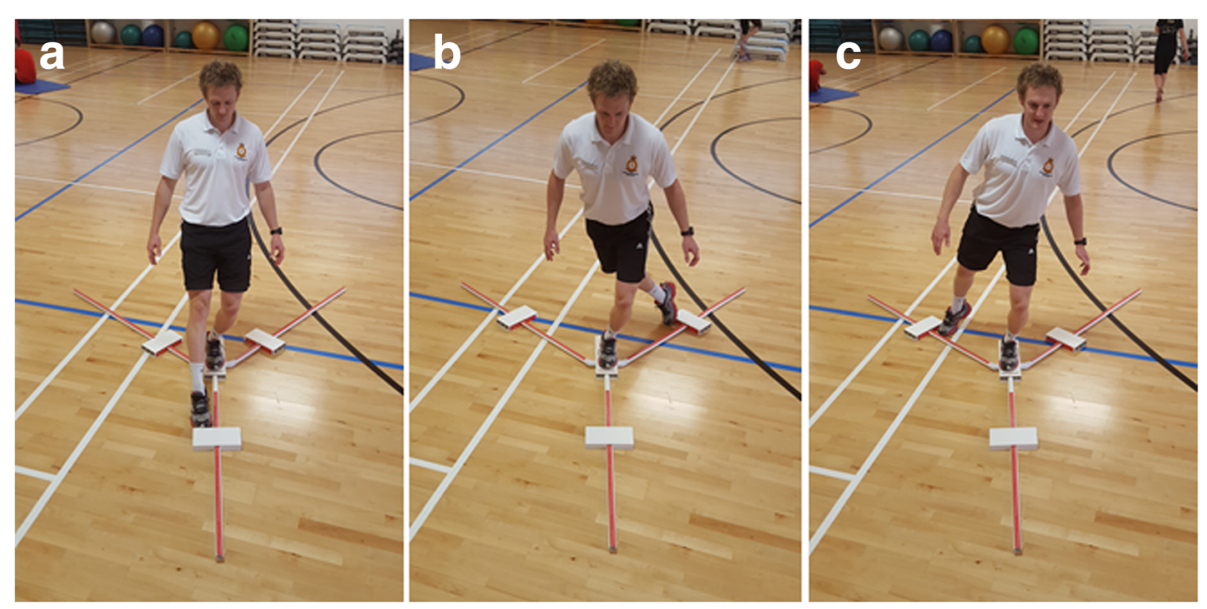

Fig. 5 Y Balance test: a anterior, b posteriomedial, c posteriolateral reach direction 
lower-limb MSK injuries. To date, this is the first study to establish the preliminary effects of LI-BFR on the muscle volume, strength measures and functional capacity of military personnel undergoing intensive, in-patient rehabilitation. Furthermore, the recruitment methods, safety, intervention adherence and acceptability of a twice daily LI-BFR intervention are essential feasibility components that need to be understood prior to embarking on a fully powered randomised controlled trial. Therefore, the findings from this feasibility study will inform a full-scale trial to determine the effectiveness of LI-BFR during in-patient, MDT rehabilitation. If feasible, military and civilian health care providers could consider LI-BFR as a cost-effective, practical rehabilitation modality to induce muscle adaptation in the absence of high mechanical loading of the lower-limb. The results of the trial will be published when they are available.

\section{Abbreviations}

BFR: Blood flow restriction; CECS: Chronic exertional compartment syndrome; CSA: Cross-sectional area; DMRC: Defence Medical Rehabilitation Centre; DMS: Defence Medical Services; ERI: Exercise Rehabilitation Instructor; GCP: Good clinical practice; LI-BFR: Low intensity blood flow restriction; MDT: Multidisciplinary team; MRI: Magnetic resonance imaging; MSK: Musculoskeletal; MSLT: Multi-stage locomotion test; MVC: Maximal voluntary contraction; PIS: Participant information sheet; RM: Repetition maximum; ROM: Range of motion; ROS: Reactive oxygen species; VAS: Visual analogue scale

\section{Acknowledgements}

The authors would like to acknowledge and thank Mr. Jakob Kristensen for developing the framework proposal and supporting ethics submission during the early stages of the study. The authors wish to thank the clinical staff on the Lower-Limbs Department of the UK Defence Medical Rehabilitation Centre (DMRC) Headley Court for supporting the conduct of this research in a busy clinical setting.

\section{Funding}

The study was funded by the UK Ministry of Defence (MOD).

\section{Availability of data and materials}

Not applicable owing to the study type.

\section{Authors' contributions}

$P L, R C, S D D$ and SDP conceived the study design. AB obtained the funding. SDD and DC will be responsible for recruiting and consenting participants into the study and delivery of the intervention. ES will analyse, interpret and report all MRI-related outcomes. PL, RC, SDD and ES will analyse the findings. $\mathrm{PL}$ and $\mathrm{RC}$ wrote the draft of the manuscript; all authors read, critically reviewed and approved the final version.

\section{Authors' information}

$\mathrm{PL}$ is the Exercise Physiology in Military Rehabilitation Research Lead, within the Academic Department of Military Rehabilitation (ADMR) at DMRC, Headley Court, UK. RC is the Clinical Research Manager on ADMR, at DMRC, Headley Court, UK. SDD is a Consultant in Rehabilitation on the Centre for Lower-Limb Rehabilitation at DMRC, Headley Court, UK. DC is a specialist Exercise Rehabilitation Instructor (ERI) at DMRC, Headley Court, UK. ES is a Defence Consultant Radiologist at Oxford University Hospital, Oxford, UK. SDP is a Senior Lecturer on the School of Sport, Health and Applied Science at St Mary's University, London, UK. AB is the Defence Professor for Rheumatology and Rehabilitation Medicine, Consultant in Rheumatology and Rehabilitation and Officer Commanding ADMR at DMRC, Headley Court, UK.

\section{Ethics approval and consent to participate}

Written informed consent will be obtained from the participant. Ethical approval was obtained for this study by the Ministry of Defence Research Ethic Committee (MODREC) (protocol number: 442/MODREC/13).

\section{Consent for publication}

Written consent was provided for all images taken within the manuscript.

\section{Competing interests}

The authors declare that they have no competing interests.

\section{Publisher's Note}

Springer Nature remains neutral with regard to jurisdictional claims in published maps and institutional affiliations.

\section{Author details}

${ }^{1}$ Academic Department of Military Rehabilitation, Defence Medical Rehabilitation Centre (DMRC), Headley Court, Epsom, Surrey, UK. ${ }^{2}$ Department for Health, University of Bath, Bath, UK. ${ }^{3}$ Imaging Department, Oxford University Hospitals, Oxford, UK. ${ }^{4}$ School of Sport, Health and Applied Science, St Mary's University, London, UK. ${ }^{5}$ National Heart and Lung Institute, Faculty of Medicine, Imperial College London, London, UK.

Received: 14 August 2017 Accepted: 1 December 2017 Published online: 08 December 2017

References

1. Seguin R, Nelson ME. The benefits of strength training for older adults. Am J Prev Med. 2003;25:141-9.

2. Slysz J, Stultz J, Burr JF. The efficacy of blood flow restricted exercise: a systematic review \& meta-analysis. J Sci Med Sport. 2016;19:669-75.

3. Hunter GR, McCarthy JP, Bamman MM. Effects of resistance training on older adults. Sports Med. 2004;34:329-48.

4. Lorenz DS, Reiman MP, Walker JC. Periodization: current review and suggested implementation for athletic rehabilitation. Sports Health. 2010;2:509-18.

5. Kristensen J, Franklyn-Miller A. Resistance training in musculoskeletal rehabilitation: a systematic review. Br J Sports Med. 2012:46:719-26.

6. American College of Sports M. American College of Sports Medicine position stand. Progression models in resistance training for healthy adults. Med Sci Sports Exerc. 2009;41:687-708.

7. Garber CE, Blissmer B, Deschenes MR, Franklin BA, Lamonte MJ, Lee IM, Nieman DC, Swain DP, American College of Sports M. American College of Sports Medicine position stand. Quantity and quality of exercise for developing and maintaining cardiorespiratory, musculoskeletal, and neuromotor fitness in apparently healthy adults: guidance for prescribing exercise. Med Sci Sports Exerc. 2011;43:1334-59.

8. Hoyt BW, Pavey GJ, Pasquina PF, Potter BK. Rehabilitation of lower extremity trauma: a review of principles and military perspective on future directions. Current Trauma Reports. 2015;1:50-60.

9. Scott BR, Loenneke JP, Slattery KM, Dascombe BJ. Exercise with blood flow restriction: an updated evidence-based approach for enhanced muscular development. Sports Med. 2015:45:313-25.

10. Takano H, Morita T, lida H, Asada K, Kato M, Uno K, Hirose K, Matsumoto A, Takenaka K, Hirata Y, et al. Hemodynamic and hormonal responses to a short-term low-intensity resistance exercise with the reduction of muscle blood flow. Eur J Appl Physiol. 2005;95:65-73.

11. Shinohara M, Kouzaki M, Yoshihisa T, Fukunaga T. Efficacy of tourniquet ischemia for strength training with low resistance. Eur J Appl Physiol Occup Physiol. 1998;77:189-91.

12. Loenneke JP, Kearney ML, Thrower AD, Collins S, Pujol TJ. The acute response of practical occlusion in the knee extensors. J Strength Cond Res. 2010;24:2831-4.

13. Loenneke JP, Fahs CA, Rossow LM, Thiebaud RS, Mattocks KT, Abe T, Bemben MG. Blood flow restriction pressure recommendations: a tale of two cuffs. Front Physiol. 2013:4:249.

14. Kubota A, Sakuraba K, Sawaki K, Sumide T, Tamura Y. Prevention of disuse muscular weakness by restriction of blood flow. Med Sci Sports Exerc. 2008; 40:529-34.

15. Scott BR, Loenneke JP, Slattery KM, Dascombe BJ. Blood flow restricted exercise for athletes: a review of available evidence. J Sci Med Sport. 2016; 19:360-7. 
16. Libardi CA, Chacon-Mikahil MP, Cavaglieri CR, Tricoli V, Roschel H, Vechin FC, Conceicao MS, Ugrinowitsch C. Effect of concurrent training with blood flow restriction in the elderly. Int J Sports Med. 2015;36:395-9.

17. Vechin FC, Libardi CA, Conceicao MS, Damas FR, Lixandrao ME, Berton RP, Tricoli VA, Roschel HA, Cavaglieri CR, Chacon-Mikahil MP, Ugrinowitsch C. Comparisons between low-intensity resistance training with blood flow restriction and high-intensity resistance training on quadriceps muscle mass and strength in elderly. J Strength Cond Res. 2015;29:1071-6.

18. Lixandrao ME, Ugrinowitsch C, Laurentino G, Libardi CA, Aihara AY, Cardoso FN, Tricoli V, Roschel H. Effects of exercise intensity and occlusion pressure after 12 weeks of resistance training with blood-flow restriction. Eur J Appl Physiol. 2015;115:2471-80.

19. Segal N, Davis MD, Mikesky AE. Efficacy of blood flow-restricted low-load resistance training for quadriceps strengthening in men at risk of symptomatic knee osteoarthritis. Geriatr Orthop Surg Rehabil. 2015;6:160-7.

20. Segal NA, Williams GN, Davis MC, Wallace RB, Mikesky AE. Efficacy of blood flow-restricted, low-load resistance training in women with risk factors for symptomatic knee osteoarthritis. PM R. 2015;7:376-84.

21. Bryk FF, Dos Reis AC, Fingerhut D, Araujo T, Schutzer M, Cury Rde P, Duarte A Jr, Fukuda TY. Exercises with partial vascular occlusion in patients with knee osteoarthritis: a randomized clinical trial. Knee Surg Sports Traumatol Arthrosc. 2016;24:1580-6.

22. Giles L, Webster KE, McClelland J, Cook JL. Quadriceps strengthening with and without blood flow restriction in the treatment of patellofemoral pain: a double-blind randomised trial. Br J Sports Med. 2017;0:1-8.

23. Tennent DJ, Hylden CM, Johnson AE, Burns TC, Wilken JM, Owens JG. Blood flow restriction training after knee arthroscopy: a randomized controlled pilot study. Clin J Sport Med. 2017;27:245-52.

24. Takarada Y, Takazawa H, Ishii N. Applications of vascular occlusion diminish disuse atrophy of knee extensor muscles. Med Sci Sports Exerc. 2000;32:2035-9.

25. Dankel SJ, Jessee MB, Abe T, Loenneke JP. The effects of blood flow restriction on upper-body musculature located distal and proximal to applied pressure. Sports Med. 2016;46:23-33.

26. Patterson SD, Brandner CR. The role of blood flow restriction training for applied practitioners: A questionnaire-based survey. J Sports Sci. 2018;36(2): 123-30.

27. Iversen E, Rostad V. Low-load ischemic exercise-induced rhabdomyolysis. Clin J Sport Med. 2010;20:218-9.

28. Tabata S, Suzuki Y, Azuma K, Matsumoto H. Rhabdomyolysis after performing blood flow restriction training: a case report. J Strength Cond Res. 2016;30:2064-8

29. Ozawa Y, Koto T, Shinoda H, Tsubota K. Vision loss by central retinal vein occlusion after Kaatsu training: a case report. Medicine (Baltimore). 2015;94:e1515.

30. Loenneke JP, Wilson JM, Wilson GJ, Pujol TJ, Bemben MG. Potential safety issues with blood flow restriction training. Scand J Med Sci Sports. 2011;21:510-8.

31. Pearson SJ, Hussain SR. A review on the mechanisms of blood-flow restriction resistance training-induced muscle hypertrophy. Sports Med. 2015;45:187-200.

32. Loenneke JP, Fahs CA, Rossow LM, Abe T, Bemben MG. The anabolic benefits of venous blood flow restriction training may be induced by muscle cell swelling. Med Hypotheses. 2012;78:151-4

33. Takarada Y, Nakamura Y, Aruga S, Onda T, Miyazaki S, Ishii N. Rapid increase in plasma growth hormone after low-intensity resistance exercise with vascular occlusion. J Appl Physiol (1985). 2000;88:61-5.

34. Reeves GV, Kraemer RR, Hollander DB, Clavier J, Thomas C, Francois M, Castracane VD. Comparison of hormone responses following light resistance exercise with partial vascular occlusion and moderately difficult resistance exercise without occlusion. J Appl Physiol (1985). 2006;101:1616-22.

35. Fujita S, Abe T, Drummond MJ, Cadenas JG, Dreyer HC, Sato Y, Volpi E, Rasmussen BB. Blood flow restriction during low-intensity resistance exercise increases 56K1 phosphorylation and muscle protein synthesis. J Appl Physiol (1985). 2007;103:903-10.

36. Fry CS, Glynn EL, Drummond MJ, Timmerman KL, Fujita S, Abe T, Dhanani S, Volpi E, Rasmussen BB. Blood flow restriction exercise stimulates mTORC1 signaling and muscle protein synthesis in older men. J Appl Physiol (1985). 2010;108:1199-209.

37. Laurentino GC, Ugrinowitsch C, Roschel H, Aoki MS, Soares AG, Neves M Jr, Aihara AY, Fernandes Ada R, Tricoli V. Strength training with blood flow restriction diminishes myostatin gene expression. Med Sci Sports Exerc. 2012:44:406-12.

38. Takarada Y, Sato Y, Ishii N. Effects of resistance exercise combined with vascular occlusion on muscle function in athletes. Eur J Appl Physiol. 2002;86:308-14.
39. Kawada S, Ishii N. Skeletal muscle hypertrophy after chronic restriction of venous blood flow in rats. Med Sci Sports Exerc. 2005;37:1144-50.

40. Pope ZK, Willardson JM, Schoenfeld BJ. Exercise and blood flow restriction. J Strength Cond Res. 2013;27:2914-26.

41. Hunt JE, Galea D, Tufft G, Bunce D, Ferguson RA. Time course of regional vascular adaptations to low load resistance training with blood flow restriction. J Appl Physiol (1985). 2013;115:403-11.

42. Casey DP, Madery BD, Curry TB, Eisenach JH, Wilkins BW, Joyner MJ. Nitric oxide contributes to the augmented vasodilatation during hypoxic exercise. J Physiol. 2010;588:373-85.

43. Hughes L, Paton B, Rosenblatt B, Gissane C, Patterson SD. Blood flow restriction training in clinical musculoskeletal rehabilitation: a systematic review and meta-analysis. Br J Sports Med. 2017;51:1003-11.

44. Andersen KA, Grimshaw PN, Kelso RM, Bentley DJ. Musculoskeletal lower limb injury risk in Army populations. Sports Med Open. 2016;2:22.

45. Sharma J, Greeves JP, Byers M, Bennett AN, Spears IR. Musculoskeletal injuries in British Army recruits: a prospective study of diagnosis-specific incidence and rehabilitation times. BMC Musculoskelet Disord. 2015;16:106.

46. Fujita T, Brechue WF, Kurita K, Sato Y, Abe T. Increased muscle volume and strength following six days of low-intensity resistance training with restricted muscle blood flow. International Journal of KAATSU Training Research. 2008:4:1-8.

47. Abe T, Yasuda T, Midorikawa T, Sato Y, Kearns CF, Inoue K, Koizumi K, Ishii N. Skeletal muscle size and circulating IGF-1 are increased after two weeks of twice daily KAATSU resistance training. International Journal of KAATSU Training Research. 2005;1:6-12.

48. Chan AW, Tetzlaff JM, Gotzsche PC, Altman DG, Mann H, Berlin JA, Dickersin K, Hrobjartsson A, Schulz KF, Parulekar WR, et al. SPIRIT 2013 explanation and elaboration: guidance for protocols of clinical trials. BMJ. 2013:346:e7586.

49. Coppack RJ, Bilzon JL, Wills AK, McCurdie IM, Partridge L, Nicol AM, Bennett AN. A comparison of multidisciplinary team residential rehabilitation with conventional outpatient care for the treatment of non-arthritic intra-articular hip pain in UK military personnel - a protocol for a randomised controlled trial. BMC Musculoskelet Disord. 2016;17:459.

50. Ageberg E, Nilsdotter A, Kosek E, Roos EM. Effects of neuromuscular training (NEMEX-TJR) on patient-reported outcomes and physical function in severe primary hip or knee osteoarthritis: a controlled before-and-after study. BMC Musculoskelet Disord. 2013;14:232.

51. Oiestad BE, Osteras N, Frobell R, Grotle M, Brogger H, Risberg MA. Efficacy of strength and aerobic exercise on patient-reported outcomes and structural changes in patients with knee osteoarthritis: study protocol for a randomized controlled trial. BMC Musculoskelet Disord. 2013;14:266.

52. Noordin S, McEwen JA, Kragh JF Jr, Eisen A, Masri BA. Surgical tourniquets in orthopaedics. J Bone Joint Surg Am. 2009;91:2958-67.

53. Reiman MP, Lorenz DS. Integration of strength and conditioning principles into a rehabilitation program. Int J Sports Phys Ther. 2011;6:241-53.

54. Linton SJ, Hellsing AL, Bergstrom G. Exercise for workers with musculoskeletal pain: does enhancing compliance decrease pain? J Occup Rehabil. 1996;6:177-90.

55. Lancaster GA, Dodd S, Williamson PR. Design and analysis of pilot studies: recommendations for good practice. J Eval Clin Pract. 2004;10:307-12.

56. Abe T, Kearns CF, Fukunaga T. Sex differences in whole body skeletal muscle mass measured by magnetic resonance imaging and its distribution in young Japanese adults. Br J Sports Med. 2003;37:436-40.

57. Abe T, Fujita S, Nakajima T, Sakamaki M, Ozaki H, Ogasawara R, Sugaya M, Kudo $\mathrm{M}$, Kurano $\mathrm{M}$, Yasuda T, et al. Effects of low-intensity cycle training with restricted leg blood flow on thigh muscle volume and VO2MAX in young men. J Sports Sci Med. 2010;9:452-8.

58. Baechle TR, Earle RW. Essentials of strength training and conditioning. 2nd ed. Human Kinetics: Champaign; 2008.

59. Thorborg K, Petersen J, Magnusson SP, Holmich P. Clinical assessment of hip strength using a hand-held dynamometer is reliable. Scand J Med Sci Sports. 2010;20:493-501.

60. Thorborg K, Bandholm T, Holmich P. Hip- and knee-strength assessments using a hand-held dynamometer with external belt-fixation are inter-tester reliable. Knee Surg Sports Traumatol Arthrosc. 2013;21:550-5.

61. Hassett LM, Harmer AR, Moseley AM, Mackey MG. Validity of the modified 20-metre shuttle test: assessment of cardiorespiratory fitness in people who have sustained a traumatic brain injury. Brain Inj. 2007; 21:1069-77. 
62. Vitale AE, Jankowski LW, Sullivan SJ. Reliability for a walk/run test to estimate aerobic capacity in a brain-injured population. Brain Inj. 1997;1 1:67-76.

63. Reiman M, Manske R. Functional testing in human performance. Champaign: Illinois:Human Kinetics; 2009.

64. Plisky PJ, Gorman PP, Butler RJ, Kiesel KB, Underwood FB, Elkins B. The reliability of an instrumented device for measuring components of the star excursion balance test. N Am J Sports Phys Ther. 2009;4:92-9.

65. Collins SL, Moore RA, McQuay HJ. The visual analogue pain intensity scale: what is moderate pain in millimetres? Pain. 1997;72:95-7.

66. Julious SA. Sample size of 12 per group rule of thumb for a pilot study. Pharm Stat. 2005:4:287-91.

Submit your next manuscript to BioMed Central and we will help you at every step:

- We accept pre-submission inquiries

- Our selector tool helps you to find the most relevant journal

- We provide round the clock customer support

- Convenient online submission

- Thorough peer review

- Inclusion in PubMed and all major indexing services

- Maximum visibility for your research

Submit your manuscript at www.biomedcentral.com/submit
Biomed Central 